\title{
Servet traditionaliste
}

\author{
Par Huldrych M. Koelbing*
}

\begin{abstract}
On ne peut parler de Michel Servet sans penser à son grand adversaire Jean CaLvin. A ce propos, la sympathie pour la victime ne devrait pas, je crois, nous faire oublier que la république calviniste de Genève, menacée de presque tous les côtés, ne fut pas seulement la «Rome protestante », mais aussi une citadelle de l'esprit d'indépendance politique, à laquelle nous devons beaucoup.
\end{abstract}

Michel Servet (1509 ou 1511-1553) qui, à cause de sa théologie, fut condamné à la mort par les protestants aussi bien que par les catholiques et à qui la médecine occidentale doit la première description de la circulation pulmonaire, est en général considéré comme un esprit révolutionnaire. C'est compréhensible et c'est justifié - mais ce n'est pas là toute la vérité: Servet était aussi profondément enraciné dans la tradition.

Dans son excellent livre Michel Servet, hérétique et martyr publié à Genève en 1953 pour commémorer le quatrième centenaire de sa mort, RoLAND $H$. Bainton a montré que les idées hétérodoxes du théologien espagnol sur la Trinité s'inspiraient des premiers Pères de l'Eglise - en particulier de TERTULLIEN et d'IrÉNÉE - et des néo-platoniciens. Les premières publications médicales de Servet - In Leonardum Fuchsium apologia et son traité sur les sirops Syruporum universa ratio - témoignent d'une connaissance profonde des autorités anciennes et médiévales, dont il acceptait toujours ce qui lui semblait le plus raisonnable. Inutile de dire que cet homme de la Renaissance se rangeait, le cas échéant, du côté des Grecs contre les Arabes.

Sa grande découverte, évidemment, est quelque chose d'original. Mais nous allons voir que, tout de même, sa conception de la circulation pulmonaire se compose essentiellement d'éléments galéniques. Pareillement, dans

* Conférence faite à la réunion annuelle de la Société suisse d'histoire de la médecine et des sciences naturelles, Genève, 25 septembre 1965. 
sa physiologie des fonctions cérébrales, nous allons retrouver des notions galéniques, aristotéliennes et bibliques. Mais tout d'abord rendons-nous compte du contexte de ces descriptions anatomo-physiologiques!

On s'étonne souvent que Servet ait décrit la circulation du sang par les poumons dans une œuvre théologique. C'est là certainement une expression de l'universalité de cet esprit qui embrassait presque toutes les connaissances humaines: la théologie et la médecine, la philosophie et la physiologie, l'astrologie et la géographie. Mais - fait important - dans cet univers spirituel, une hiérarchie régnait qu'on pourrait qualifier de médiévale: tout était subordonné à la théologie. Servet étudiait l'homme pour mieux comprendre Dieu; il exposait la physiologie pour illustrer la sagesse et la bonté divines. Par cette attitude, il ressemble à Galien qui, lui aussi, dans son œuvre sur L'utilité des parties $d u$ corps, glorifie sans cesse la perfection et la providence divines. Les différences, pourtant, sont évidentes: le Créateur de Galien est identique à la Nature, déité tout à fait impersonnelle; d'autre part, le Dieu de Servet est bien le Dieu de la Bible qui entre en communication personnelle avec les hommes. Et malgré ses intentions philosophiques, De usu partium reste un traité systématique de physiologie, tandis que les dix pages ${ }^{1}$ que Servet consacre aux explications anatomiques et physiologiques ne changent en rien le caractère purement théologique de sa Restitution du christianisme. Pour cette raison, si nous voulons comprendre la physiologie de Servet, nous ne pouvons négliger complètement sa théologie.

\section{II}

Pour Servet théologien, le problème central était celui de la Trinité. Déjà le titre de la première partie de Christianismi restitutio esquisse la conception qu'il s'en était faite: «De la Trinité divine, qu'en elle il n'y a pas illusion de trois choses invisibles, mais la vraie manifestation de la substance de Dieu dans le Verbe et sa communication dans l'Esprit.» (De trinitate divina, quod in ea non sit invisibilium trium rerum illusio, sed vera substantiae Dei manifestatio in verbo et communicatio in spiritu.)

Mais ce n'est là que le noyau d'un système religieux bien mystique: Dieu - quoiqu'il soit au-delà de tout ce qui peut être conçu - est présent dans tout ce qu'il a créé, dans la pierre et le bois par exemple, et à plus forte raison dans l'âme humaine: en créant l'homme, «Dieu introduit l'âme pro-

1 Christianismi restitutio [Vienne en Dauphiné] 1553, p. 169-178. Réimpression [Nuremberg] 1790, pagination identique. 
venant de l'air; avec l'âme même, il introduit l'air et une étincelle de la Déité même qui remplit l'air» (p. 168). Autrement dit: l'air élémentaire, que nous respirons, est imprégné de l'esprit saint de Dieu.

Mais la création ne fut pas qu'un événement historique et unique, c'est au contraire un processus qui se perpétue, en quelque sorte, dans la respiration. Pour le croyant, la respiration peut redevenir l'inspiration qui lui ouvre le chemin de la régénération l'amenant à l'union mystique avec Dieu et le Christ. Ainsi, il n'existe pas, pour Servet, de limite nette entre la vie spirituelle et la vie physique de l'homme. Tout au contraire: sans une vie spirituelle, quelque rudimentaire qu'elle soit, la vie physique est impensable. Ces rapports mystiques entre Dieu et sa créature, l'esprit divin et l'âme humaine, sont examinés dans le Cinquième livre de la Trinité divine, traitant du Saint-Esprit (p. 163). Pour commencer, Servet répète le fondement de sa croyance: «L'essence de Dieu, en tant qu'elle se manifeste au monde, est le Verbe; en tant qu'elle se communique au monde, elle est l'Esprit» (p. 163). La parole et l'esprit, voire l'haleine de l'homme sont les images de la parole et l'esprit de Dieu. Mais ce n'est pas une simple similitude - nous le savons déjà : d'une manière mystérieuse, Dieu lui-même est présent dans l'esprit, l'âme et le souffle de l'homme. Cette présence de Dieu dans les hommes a été particulièrement renforcée par l'apparition du Christ: «Par le Christ, la créature est unie au créateur... dans la chair aussi bien que dans l'esprit; elle ne possède qu'une substance avec Dieu (est... hypostatice unum cum Deo). Voici le grand mystère et notre union substantielle avec le Père par le Fils » (p. 166). On pourrait peut-être traduire cette pensée de Servet comme suit: le Christ intensifie la communion, préexistante en puissance, entre Dieu et l'homme.

\section{III}

C'est pour établir les bases naturelles de cette union mystique de Dieu et de l'homme que Servet a recours à l'anatomie et à la physiologie. Il part de quelques données établies dans l'Ancien Testament: «Ezéchiel enseigne que l'âme tient quelque chose de la substance élémentaire; qu'elle tienne quelque chose de la substance du sang, Dieu lui-même l'enseigne [par Moïse] ... Pour te donner un exposé complet et systématique de l'âme et de l'esprit (ut vero totam animae et spiritus rationem habeas), ô lecteur, je vais ajouter ici cette philosophie divine que tu comprendras facilement si tu es exercé en anatomie» (p. 169). On dit qu'il y a en nous un triple esprit - naturel, vital et animal - formé des trois éléments supérieurs - l'eau, l'air et le feu. Mais 
en réalité, ce n'est qu'un esprit, l'esprit vital, dont provient le naturel aussi bien que l'animal. Le seul esprit de Dieu et sa lumière donnent à tous les trois leur activité.

L'esprit vital, qui réside dans le cœur et les artères, peut pénétrer dans les veines grâce aux anastomoses qui réunissent les deux systèmes vasculaires ce sont les anastomoses d'Erasistrate. Aussitôt entré dans les veines, l'esprit vital prend le nom d'esprit naturel. Il entre dans la composition du sang veineux; Servet dit même qu'il est le sang veineux (primus ergo [sc. spiritus] est sanguis, cuius sedes est in hepate et corporis venis, p. 169), et on a voulu conclure de cette seule phrase que Servet avait une idée de la circulation générale du sang ${ }^{2}$; mais la suite de son exposé nous convainc du contraire.

La destination essentielle de l'esprit vital, c'est de former la substance de l'âme. «Il est produit dans les poumons par un mélange d'air inspiré et de sang subtil, élaboré, communiqué par le ventricule droit du cour au ventricule gauche» (p. 170). Et maintenant, Servet décrit le trajet de ce sang - il ne s'agit, bien entendu, que d'une fraction de la masse totale du sang formé par le foie - du ventricule droit du cœur à travers les poumons et retour au ventricule gauche. Il étaye sa théorie de deux observations anatomiques: L'artère pulmonaire (vena arteriosa) est beaucoup trop large pour n'être destinée qu'à la nutrition des poumons, et le septum cardiaque ne possède pas de vaisseaux ni d'autres moyens d'assurer le passage du sang d'un ventricule à l'autre. «Il est toutefois possible que quelque chose y passe par transsudation» (licet aliquid resudare possit, p. 171). A ces données morphologiques Servet ajoute une observation d'ordre fonctionnel: ce n'est pas le cœur, mais les poumons qui donnent au sang spiritueux sa coloration «jaune» (flavus ille color, p. 171). L'esprit vital, formé ainsi d'air et de sang raffiné, constitue pour ainsi dire la substance de l'âme même: «hoc totum veluti materia est ipsius animae» (p. 178).

Les arguments avancés par Servet en faveur de la circulation pulmonaire ne laissent guère douter qu'il a vraiment observé, de ses propres yeux, ce qu'il décrit. Certainement, il a étudié les vaisseaux pulmonaires sur des cadavres humains, peut-être aussi sur un animal vivant. Tout de même, sa conception s'écarte bien moins des enseignements de Galien qu'on ne le croit en général. Comme L.G. Wilson le souligne ${ }^{3}$, Galien a déjà noté que le sang artériel, par comparaison avec le sang veineux, est "plus jaune»

2 H.ToluIn, Die Entdeckung des Blutkreislaufes durch Michael Servet, Jena 1876.

${ }^{3}$ L. G. WiLson, Erasistratus, Galen and the pneuma, Bull. Hist.Med. 33 (1959) 293. 
( $\xi a v \vartheta o ́ \tau \varepsilon \varrho o v$, flavior - De placitis Hippocratis et Platonis, VI/4). Servet ne prétend pas que le septum cardiaque est absolument imperméable au sang, alors qu'aussi pour Galien, les anastomoses entre artères et veines, au niveau du poumon, sont d'une importance physiologique considérable: par ces conduits étroits, le système veineux - représenté ici par les ramifications de l'artère pulmonaire - reçoit du pneuma; en revanche, un peu de sang les traverse dans le sens opposée ${ }^{4}$. Une circulation pulmonaire minime était donc admise par Galien, et on peut dire - avec $0^{\text {'MALLEY }}{ }^{5}$ - que Servet a simplement renversé les proportions du sang passant par le septum cardiaque d'une part, par les poumons d'autre part. Mais ce renversement des proportions était le début d'une physiologie nouvelle, basée sur une connaissance approfondie des corps humain et animal.

\section{IV}

Arrivés à ce point, les médecins désireux d'apprécier la contribution de Servet à la physiologie de la circulation sanguine s'arrêtent en général. Mais si nous voulons avoir une idée d'ensemble de sa physiologie, ou disons plutôt: de son anthropologie, il nous faut le suivre dans la discussion des fonctions cérébrales. L'esprit vital est distribué par les artères dans le corps tout entier, mais sa portion la plus fine monte vers la tête, passe par le plexus rétiforme situé, selon les enseignements de Galien, à la base du cerveau et pénètre enfin dans les vaisseaux extrêmement minces des plexus choroïdes. Ces vaisseaux - ce sont des «artères capillaires 》 - constituent la résidence de l'intellect même - «ipsissimam mentem continent» (p. 171), et c'est le feu de celui-ci qui achève une nouvelle élaboration de l'esprit vital: sa transformation en esprit animal.

Et maintenant, encore une fois, Servet s'écarte décidément de la doctrine galénique: ces petits vaisseaux capillaires des plexus choroïdes relient le système artériel aux nerfs, la liaison s'effectuant par l'intermédiaire de la pie-mère. Nous sommes en présence d'un nouveau genre de vaisseaux: comme il existe dans les poumons un genre nouveau de vaisseaux pour le passage du sang des veines aux artères, il existe ici un genre nouveau de vaisseaux pour le transport de l'esprit des artères aux nerfs (p. 172).

${ }^{4}$ Galien, De usu partium, VI/10, dans Ch. Daremberg, Euvres de Galien, t. I, Paris 1854, p. 414.

5 CH.D. O'Malley, The complementary careers of Michael Servetus ..., J.Hist. Med. 8 (1953) 378. 
Suivant la tradition galénique, les plexus choroïdes sécrétaient l'esprit animal dans les ventricules du cerveau ${ }^{6}$. De là, il pénétrait dans la substance cérébrale qui, à son tour, le distribuait aux nerfs. Il semble que, pour Servet théologien, cette conception n'ait pas été admissible. Comme l'âme est dans le sang, et le sang dans les vaisseaux, les esprits ne doivent pas sortir des systèmes vasculaires, parmi lesquels il faut, bien entendu, compter aussi les nerfs. Le moyen de connaître les voies invisibles de communication entre les systèmes artériel et nerveux était pour Servet l'analogisme, employé si libéralement par les Anciens: ce qui était valable pour les poumons devait être valable aussi pour le cerveau. Mais pour la circulation pulmonaire, Servet pouvait s'appuyer sur de bonnes observations anatomiques; par contre, en postulant l'existence des communications artério-nerveuses au niveau du cerveau, il était contraint de négliger un fait bien connu de Galien, à savoir que les nerfs crâniens tirent leur origine de la substance cérébrale et non pas des méninges.

Quant au cerveau, quelle fonction Servet pouvait-il lui attribuer? Il est évident que, pour répondre à cette question, il avait recours à Aristote, qui lui aussi avait nié le rôle central et dominant du cerveau dans la vie psychique et intellectuelle et qui en avait fait un organe réfrigérateur ( $D e$ partibus animalium II/7). Comme Aristote, Servet maintient que la masse cérébrale, insensible, ne peut être le siège de l'âme raisonnable. Mais Servet pense qu'elle peut servir, à l'instar d'un coussin, de support aux petits vaisseaux de l'esprit. En outre, par sa froideur, elle est propre à tempérer la chaleur ardente de celui-ci.

Il lui était plus facile d'expliquer l'utilité des ventricules cérébraux. Même si l'esprit animal en était banni, il leur restait encore deux de leurs fonctions traditionnelles: c'étaient à la fois une espèce de cloaques destinés à recevoir les excréments du cerveau et des récipients pour cette partie de l'air inspiré qui entrait du nez directement dans la cavité crânienne, à travers les éthmoïdes. Servet dit que cet air rafraîchit et évente le contenu des «vaisseaux de l'âme», constitué par l'intellect (mens), l'âme (anima) et un esprit ardent (igneus spiritus, p. 173). Ce dernier doit être identique à l'esprit animal, qui est le substratum des activités intellectuelles et psychiques; mais il vaut certainement mieux de ne pas trop insister sur les détails de cette matière très difficile... Retenons simplement que, pour Servet, l'acti-

6 Cf. K. E. Rотнsснuн, Vom Spiritus animalis zum Nervenaktionsstrom, Ciba-Z. 8 (Wehr/ Baden) (Nr. 89) (1958) 2950. 
vité psychique est une espèce de combustion, qui a besoin du sang comme combustible aussi bien que de l'esprit contenu dans l'air respiré. C'est donc une combustion matérielle et spirituelle à la fois.

C'est vers le même endroit, c'est-à-dire les anastomoses artério-nerveuses au niveau des plexus choroïdes, que le Saint-Esprit se dirige - mais aussi son adversaire, l'esprit malin provenant des abîmes des ventricules cérébraux. Ces petits vaisseaux profondément cachés peuvent être le lieu de révélations divines, mais aussi le point d'origine d'une «fureur démoniaque». A part ces événements spectaculaires, une lutte continuelle se déroule, dans ces conduits étroits, entre l'esprit malin et notre propre esprit qui - vous le savez - est en principe de nature divine.

Naturellement, le cœur est aussi associé à ce combat du bien et du mal: il est la résidence de la volonté et de la charité, mais aussi du péché original.

Ainsi, la physiologie de Servet débouche à nouveau dans la théologie - et dans la philosophie: encore plus clairement que l'Ecriture sainte, Platon a constaté, dans le Timée, que «la substance de l'âme est un certain mélange de deux substances, l'une élémentaire et l'autre divine» (p. 178). De là, Servet repart pour de longues discussions sur la nature et l'activité du Saint-Esprit. Mais pour nous, il suffit d'avoir vu comment ce théologienmédecin envisage l'homme, formé de matières élémentaires et d'esprit divin à la fois.

L'étude de la physiologie de Servet - étude qu'on pourrait encore compléter par maints détails - nous a montré qu'on ne peut, chez lui, établir une distinction entre le médecin et le théologien. C'est le même esprit indépendant qui réfute le dogme officiel de la Trinité et qui corrige Galien en découvrant la circulation pulmonaire. Mais malgré l'apparence révolutionnaire de ses idées très personnelles, cet homme vit essentiellement de la tradition, antique et médiévale, qu'il a fait sienne dans une mesure tout à fait étonnante. Pour en revenir à la physiologie, la découverte de la circulation pulmonaire fut moins une révolution que le résultat d'une longue évolution au sein de la médecine traditionnelle et galénique. 

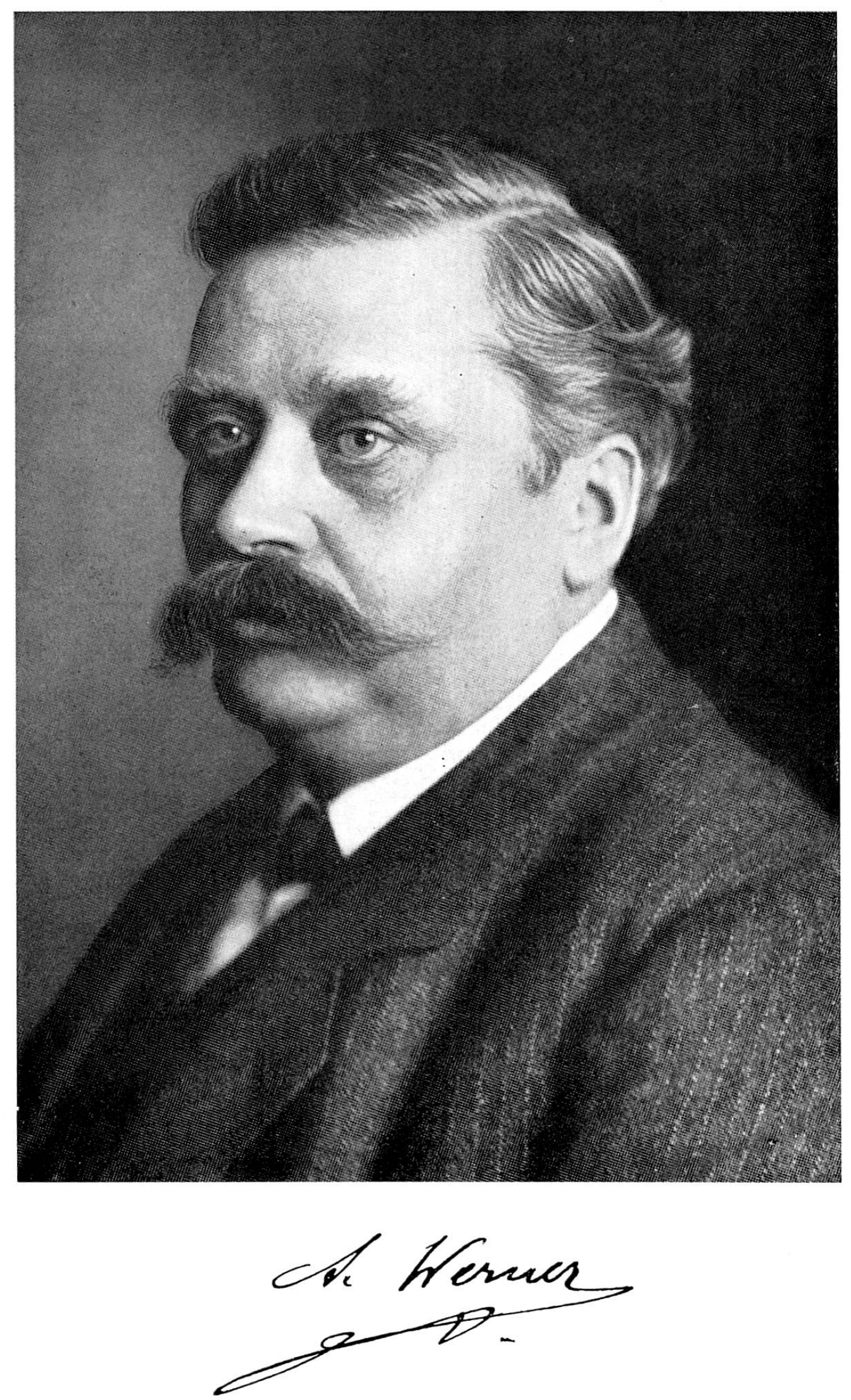


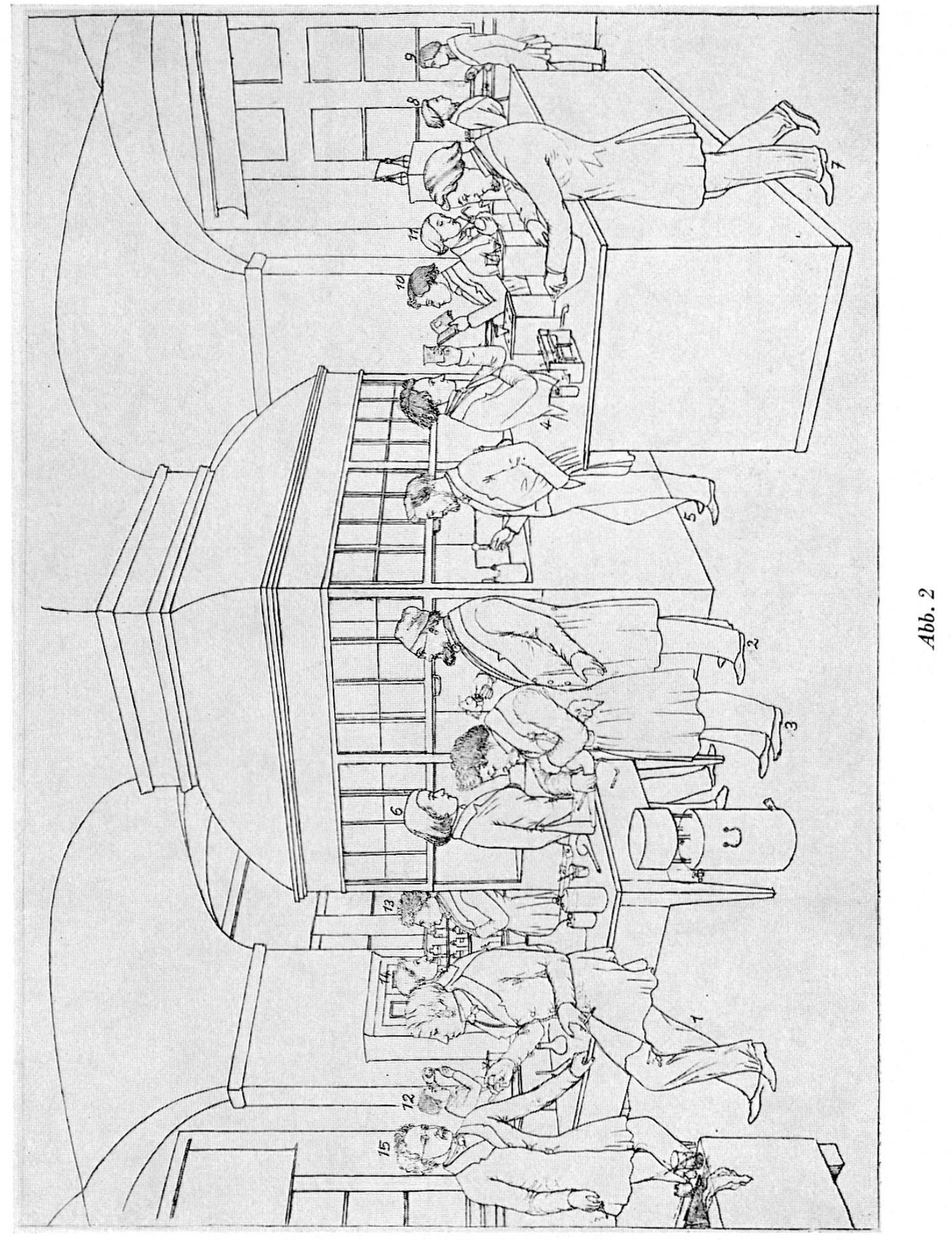

